\title{
Encuesta nacional sobre tratamiento quirúrgico de patología del hígado, vía biliar y páncreas*
}

\author{
Drs. JUAN HEPP K. ${ }^{1}$, CARLOS BENAVIDES C. ${ }^{2}$
}

1 Past Presidente.

2 Presidente Departamento Cirugía Hígado, Vía Biliar y Páncreas. Sociedad de Cirujanos de Chile.

Santiago, Chile.

\begin{abstract}
An enquiry about liver, biliary and pancreatic surgery in Chile

Background: Chile has a high incidence of cholelithiasis and associated complications, that usually require surgical treatment. Aim: To carry out an enquiry about biliary diseases and their surgical treatment in public and private surgical services in Chile. Material and Methods: An enquiry about liver and biliary diseases and their treatment was designed and sent by electronic mail to 35 surgical services. Results: The enquiry was answered by 17 services. A total of 9.505 cholecystectomies were reported. Between 21 and $98 \%$ of the procedures were laparoscopic. Iatrogenic lesions of the biliary tree were reported in $0.3 \%$ of the procedures. Choledocolithiasis was found associated to cholelithiasis in 0 to $21 \%$ of procedures. However, the use of intraoperative cholangiography was incidental. The incidence of gallbladder cancer in cholecystectomies for cholelithiasis fluctuated between 0.2 and $8.9 \%$. Liver metastases derived from colorectal cancer were the most common liver tumor operated. Hydatidosis was more common in southern Chile. Pancreatic excision was almost exclusively performed in Metropolitan Santiago. Conclusions: This information will allow the establishment of information and collaboration channels among the surgical services throughout Chile.
\end{abstract}

Key words: Enquiry, biliary surgery, pancreatic surgery, cholecystectomy.

\section{Resumen}

Se trata de una encuesta realizada con el objeto de conocer la frecuencia e incidencia de las principales patologías y técnicas quirúrgicas de la esfera hepato-bilio-pancreática atendida en centros de salud públicos y privados del país durante el año 2007. La encuesta fue respondida por 17 instituciones nacionales. Entre los resultados resaltan el total de colecistectomías reportadas de 9.505 , de las cuales entre un 21 y $98 \%$ son realizadas por vía laparoscópica. Lesiones iatrogénicas de vía biliar ocurrieron dentro de un rango de 0 a $1,5 \%$, con una mediana de $0,3 \%$. La frecuencia de coledocolitiasis asociada a la colelitiasis ocurrió entre un 0 a $21 \%$, aunque el empleo de colangiografía intraoperatoria es en general de ocurrencia incidental. La incidencia de cáncer vesicular en colecistectomías por colelitiasis es claramente distinta según se trate de instituciones de centro-norte y sur del país, con incidencias que varían entre 0,2 y $8,9 \%$. Las metástasis hepáticas operadas son principalmente de origen colorrectal, y son los tumores hepáticos más frecuentemente tratados. La hidatidosis hepática manifiesta en esta encuesta su conocida distribución geográfica, siendo más frecuentemente tratada en los centros del sur del país. La cirugía pancreática resectiva tiende a estar concentrada en las instituciones de la región metropolitana.

Palabras clave: Encuesta, biliar, hígado, páncreas, cirugía.

*Recibido el 20 de Octubre de 2009 y aceptado para publicación el 2 de Diciembre de 2009.

Correspondencia: Dr. Juan Hepp K.

Vitacura 5951, Santiago, Chile.

E-mail: jhepp@alemana.cl 


\section{Introducción}

Son conocidas las particulares características epidemiológicas nacionales con respecto a las enfermedades hépato-bilio-pancreáticas. La alta frecuencia nacional de colelitiasis y sus complicaciones, nos hace constituir una población de características particulares y que manifiesta comportamientos clínicos distintos a lo reportado en otras regiones, como por ejemplo las de origen anglosajón. De más está mencionar nuestra alta incidencia y tasa de mortalidad femenina por cáncer vesicular, lo cual es citado frecuentemente por diversos autores extranjeros al referirse al tema.

Estas particularidades nacionales hacen del todo necesario contar con la información estadística de frecuencia e incidencia de patologías o terapias quirúrgicas utilizadas, para la discusión, investigación y conocimiento actualizado de las principales patologías hépato-bilio-pancreáticas nacionales. Por este motivo durante el año 2006 bajo el alero de la Sociedad de Cirujanos de Chile, se organizó el "Departamento de Cirugía de Hígado, Vía Biliar y Páncreas", que tiene por objetivo central promover la investigación nacional, con énfasis en trabajo cooperativo, que incremente el conocimiento sobre nuestra realidad en esta área de las enfermedades digestivas.
Con el objeto de conocer la frecuencia, incidencia y características de las principales enfermedades hépato-bilio-pancreáticas en Chile, realizamos una encuesta a los servicios quirúrgicos de hospitales públicos y clínicas privadas del país, de manera de lograr una muestra nacional representativa y actualizada sobre estas patologías. Se trata también de dar inicio a una red de cooperación inter institucional que facilite la investigación cooperativa a través de este nuevo Departamento de la Sociedad de Cirujanos de Chile.

\section{Material y Método}

Se confeccionó una encuesta que preguntaba sobre las principales características de las patologías hépato-bilio-pancreáticas atendidas por los diversos centros públicos y privados durante el año 2007.

La encuesta fue enviada mediante correo electrónico a 35 centros nacionales logrando respuesta de 17 de ellos, tanto de Santiago como de Regiones. Los centros que participaron y los cirujanos que recolectaron y enviaron los datos, aparecen citados en la Tabla 1.

Los datos fueron tabulados por los autores usando denominaciones geográficas y según se trate de

Tabla 1. Instituciones y responsables que contestaron la encuesta

\begin{tabular}{ll}
\hline Hospital de Coquimbo & Eduardo Coddou \\
Hospital San Juan de Dios Los Andes & Jaime Fischer \\
Hospital Gustavo Fricke de Viña del Mar & JE Carter, Verónica Silva \\
Hospital de Quillota & Oriana Valenzuela \\
Clínica Alemana de Santiago & Juan Hepp \\
Hospital Clínico Universidad Católica & Nicolás Jarufe \\
Clínica Dávila & Sergio Álvarez \\
Fundación Arturo López Pérez & Hernán de la Fuente \\
Hospital Clínico de la Universidad de Chile & Attila Csendes \\
Hospital San Juan de Dios & José M. Palacios \\
Hospital Clínico San Borja-Arriarán & Carlos Benavides \\
Hospital de Talca & Jorge Llanos \\
Sanatorio Alemán de Concepción & Franco Innocenti \\
Hospital Regional de Concepción & Ricardo Durán \\
Hospital Regional de Temuco & Luis Burgos \\
Hospital de Valdivia & Carlos Cárcamo \\
Hospital de Coyhaique & Félix Torres \\
\hline
\end{tabular}


centros públicos y privados, con el objeto de evitar comparaciones e interpretaciones que pudieran ser indeseables. Los hospitales universitarios se consideraron como centros privados.

\section{Resultados}

El primer rubro de la encuesta se refirió a diversos aspectos sobre litiasis biliar. El número de colecistectomías realizadas por todas las instituciones durante el año 2007 fue de 9.505 intervenciones (Tabla 2). El porcentaje de las colecistectomías realizadas laparoscópicamente tiene un rango de 21 a $98 \%$ con una mediana de $80 \%$. En cuatro instituciones este porcentaje es menor al $60 \%$. La colangiografía intraoperatoria rutinaria es realizada entre 0 a $96 \%$, con mediana de $10 \%$ en el caso de colecistectomías laparoscópicas y $15 \%$ en el caso de colecistectomías por laparotomía.

El porcentaje reportado de coledocolitiasis asociada a colelitiasis tiene un rango de 0 a $21 \%$ con mediana de $10 \%$. La lesión iatrogénica de vía biliar durante colecistectomía laparoscópica ocurrió en un rango de 0 a 1,5\%, con mediana de $0,3 \%$.

El segundo rubro se refirió al cáncer vesicular y hepático. La incidencia de cáncer vesicular encontrado en colecistectomías por colelitiasis presentó un rango que oscila entre 0,2 y $8,9 \%$, con una mediana de $2,8 \%$. Como es ya conocido, la mayor incidencia de cáncer vesicular corresponde a instituciones del sur. El número de cánceres vesiculares tratados durante el año 2007 varió entre 2 y 98 casos, y los casos resecados oscilaron entre $8 \%$ y $100 \%$ (Tabla 3). El número de carcinomas hepatocelulares y colangiocarcinomas tratados por cada institución es en general bajo, oscilando entre 0 y 7 casos (Tabla 4).

Con respecto a las metástasis
Tabla 2. Colecistectomías

\begin{tabular}{lcc}
\hline & n colecistectomías & \% laparoscópicas \\
\hline Centro Norte & 423 & 86 \\
Centro Norte & 356 & 71 \\
Centro Norte & 619 & 60 \\
Centro Norte & 399 & 59 \\
Metropolitana privado & 421 & 92 \\
Metropolitana privado & 820 & 89 \\
Metropolitana privado & 923 & 97 \\
Metropolitana privado & 50 & 80 \\
Metropolitana privado & 1.170 & -- \\
Metropolitana público & 520 & 43 \\
Metropolitana público & 602 & 93 \\
Sur & 476 & 88 \\
Sur & 282 & 94 \\
Sur & 1.103 & 98 \\
Sur & 598 & 21 \\
Sur & 421 & 75 \\
Sur & 322 & 80 \\
& 4.505 & 80 \\
& total &
\end{tabular}

Tabla 3. Cáncer vesicular

\begin{tabular}{|c|c|c|c|}
\hline & $\begin{array}{c}\text { \% cáncer en } \\
\text { colecistectomías }\end{array}$ & $\begin{array}{c}\text { Casos } \\
\text { tratados }\end{array}$ & $\begin{array}{l}\text { Cánceres } \\
\text { resecados }\end{array}$ \\
\hline Centro Norte & 2,4 & 10 & 2 \\
\hline Centro Norte & 2,8 & 10 & 6 \\
\hline Centro Norte & 2,3 & 14 & 1 \\
\hline Centro Norte & 3,5 & 14 & 10 \\
\hline Metropolitana privado & 1,0 & 4 & 1 \\
\hline Metropolitana privado & 0,2 & 2 & 2 \\
\hline Metropolitana privado & 0,5 & 5 & 4 \\
\hline Metropolitana privado & -- & 37 & 27 \\
\hline Metropolitana privado & 2,5 & 29 & 24 \\
\hline Metropolitana público & 5,2 & 27 & 4 \\
\hline Metropolitana público & 2,3 & 18 & 12 \\
\hline Sur & -- & -- & -- \\
\hline Sur & 0,7 & 2 & 1 \\
\hline Sur & 8,9 & 98 & 67 \\
\hline Sur & 0,3 & 2 & 2 \\
\hline Sur & 7,1 & 30 & 11 \\
\hline Sur & -- & -- & -- \\
\hline mediana & 2,8 & 14 & 6 \\
\hline total & & 314 & 174 \\
\hline
\end{tabular}


Tabla 4. Cáncer del hígado

\begin{tabular}{lcc}
\hline & $\begin{array}{c}\text { Carcinoma } \\
\text { hepatocelular }\end{array}$ & $\begin{array}{c}\text { Colangiocar- } \\
\text { cinoma }\end{array}$ \\
\hline Centro Norte & 1 & 0 \\
Centro Norte & 0 & 0 \\
Centro Norte & 2 & 0 \\
Centro Norte & 0 & 0 \\
Metropolitana privado & 0 & 0 \\
Metropolitana privado & 4 & 2 \\
Metropolitana privado & 2 & 1 \\
Metropolitana privado & 5 & 7 \\
Metropolitana privado & 7 & 2 \\
Metropolitana público & 1 & 0 \\
Metropolitana público & 5 & 3 \\
Sur & 0 & 0 \\
Sur & 0 & 2 \\
Sur & 4 & 3 \\
Sur & 0 & 0 \\
Sur & 1 & 0 \\
Sur & 0 & 0 \\
Total & 32 & 20 \\
\hline
\end{tabular}

Tabla 6. Quistes Hidatídicos hepáticos

\begin{tabular}{lc}
\hline & n \\
\hline Centro Norte & 4 \\
Centro Norte & 3 \\
Centro Norte & 3 \\
Centro Norte & 0 \\
Metropolitana privado & 0 \\
Metropolitana privado & 2 \\
Metropolitana privado & 2 \\
Metropolitana privado & 0 \\
Metropolitana privado & 10 \\
Metropolitana público & 2 \\
Metropolitana público & 4 \\
Sur & 17 \\
Sur & 2 \\
Sur & 24 \\
Sur & 23 \\
Sur & 11 \\
Sur & 25 \\
\hline
\end{tabular}

Tabla 5. Metástasis hepáticas

\begin{tabular}{lccc}
\hline & $\begin{array}{c}\text { Colorrec- } \\
\text { tales }\end{array}$ & $\begin{array}{c}\text { Otros } \\
\text { sitios }\end{array}$ & Total \\
\hline Centro Norte & 2 & 0 & 2 \\
Centro Norte & 1 & 0 & 1 \\
Centro Norte & 6 & 3 & 9 \\
Centro Norte & 0 & 0 & 0 \\
Metropolitana privado & 8 & 0 & 8 \\
Metropolitana privado & 16 & 3 & 19 \\
Metropolitana privado & 6 & 0 & 6 \\
Metropolitana privado & 25 & 10 & 35 \\
Metropolitana privado & 8 & 3 & 11 \\
Metropolitana público & 1 & 0 & 1 \\
Metropolitana público & 7 & 2 & 9 \\
Sur & 0 & 0 & 0 \\
Sur & 5 & 1 & 6 \\
Sur & 6 & 2 & 8 \\
Sur & 0 & 0 & 0 \\
Sur & 3 & 2 & 5 \\
Sur & 0 & 0 & 0 \\
total & 94 & 26 & 120 \\
mediana & 5 & 6 \\
\hline
\end{tabular}

hepáticas tratadas durante el año 2007, las más frecuentes fueron aquellas de origen colorrectal, con una frecuencia que va entre 0 y 25 casos. El número total de metástasis tratadas por todas las instituciones durante el año en estudio fue de 120 casos (Tabla 5).

Los quistes hidatídicos operados oscilan entre $0 \mathrm{y}$ 25 casos, siendo una patología que se ve fundamentalmente en las instituciones del sur. Sólo un centro metropolitano atendió más de cinco casos durante el año 2007 (Tabla 6).

Otras enfermedades hepáticas como quistes benignos, hemangiomas, tumores benignos del hígado, enfermedad de Caroli, son de baja frecuencia y aparecen en la Tabla 7.

El número de traumatismos hepáticos varían entre 0 y 15 casos por año, con una mediana de 2 por institución (Tabla 8).

La cirugía pancreática está expresada en la Tabla 9.

Cuatro centros que respondieron la encuesta realizaron trasplante hepático durante el año 2007, siendo todos del área privada y de la Región Metropolitana (Tabla 10). 
Tabla 7. Hígado: resecciones misceláneas

\begin{tabular}{lcccc}
\hline & Hemangioma & Quiste simple & Otros Tu. benigno & Caroli \\
\hline Centro Norte & 0 & 2 & 0 & 0 \\
Centro Norte & 0 & 0 & 0 & 0 \\
Centro Norte & 0 & 0 & 0 & 0 \\
Centro Norte & 0 & 1 & 0 & 0 \\
Metropolitana privado & 0 & 4 & 2 & 1 \\
Metropolitana privado & 2 & 3 & 1 & 5 \\
Metropolitana privado & 0 & 1 & 1 & 1 \\
Metropolitana privado & 0 & 0 & 0 & 0 \\
Metropolitana privado & 0 & 0 & 0 & 3 \\
Metropolitana público & 0 & 0 & 1 & 2 \\
Metropolitana público & 0 & 1 & 2 & 2 \\
Sur & 0 & 0 & 0 & 0 \\
Sur & 0 & 0 & 0 & 0 \\
Sur & 3 & 5 & 3 & 2 \\
Sur & 1 & 0 & 0 & 0 \\
Sur & 0 & 3 & 2 & 1 \\
Sur & 0 & 0 & 0 & 0 \\
& 6 & 20 & 12 & 17 \\
\hline
\end{tabular}

Tabla 8. Trauma hepático

\begin{tabular}{lc}
\hline & n \\
\hline Centro Norte & 7 \\
Centro Norte & 3 \\
Centro Norte & 15 \\
Centro Norte & 2 \\
Metropolitana privado & 5 \\
Metropolitana privado & 0 \\
Metropolitana privado & 2 \\
Metropolitana privado & 0 \\
Metropolitana privado & 0 \\
Metropolitana público & 12 \\
Metropolitana público & 0 \\
Sur & 0 \\
Sur & 1 \\
Sur & 7 \\
Sur & 1 \\
Sur & 12 \\
Sur & 68 \\
\hline
\end{tabular}

Tabla 9. Cirugía pancreática

\begin{tabular}{|c|c|c|c|}
\hline & $\begin{array}{l}\text { Pancreatoduo- } \\
\text { denectomía }\end{array}$ & $\begin{array}{l}\text { Paliación } \\
\text { cáncer }\end{array}$ & $\begin{array}{c}\text { Pancreat. } \\
\text { distal }\end{array}$ \\
\hline Centro Norte & 1 & 2 & 1 \\
\hline Centro Norte & 1 & 0 & 4 \\
\hline Centro Norte & 20 & 5 & 3 \\
\hline Centro Norte & 0 & 0 & 0 \\
\hline Metropolitana privado & 9 & 3 & 13 \\
\hline Metropolitana privado & 12 & 10 & 15 \\
\hline Metropolitana privado & 1 & 1 & 3 \\
\hline Metropolitana privado & 23 & 14 & 13 \\
\hline Metropolitana privado & 24 & 8 & 6 \\
\hline Metropolitana público & 6 & 6 & 3 \\
\hline Metropolitana público & 10 & 8 & 8 \\
\hline Sur & 3 & 0 & 0 \\
\hline Sur & 2 & 8 & 4 \\
\hline Sur & 12 & 19 & 2 \\
\hline Sur & 0 & 0 & 1 \\
\hline Sur & 8 & 6 & 4 \\
\hline Sur & 0 & 0 & 0 \\
\hline Total & 132 & 90 & 80 \\
\hline
\end{tabular}


Tabla 10. Trasplante hepático

\begin{tabular}{lc}
\hline & n \\
\hline Metropolitana privado & 15 \\
Metropolitana privado & 20 \\
Metropolitana privado & 2 \\
Metropolitana privado & 18 \\
\hline
\end{tabular}

\section{Discusión}

Para todos es conocida la dificultad que existe en nuestro medio para generar trabajos cooperativos y/o participación colectiva, la cual se ve expresada en la baja respuesta a esta sencilla encuesta. A pesar de todo, logramos reunir un número importante de casos e instituciones, que reflejan en cierta forma la realidad nacional con respecto a la frecuencia de las enfermedades hepato-bilio-pancreáticas.

Esta serie resume un importante número de colecistectomías (9.505 intervenciones) que sin duda representa nuestra alta frecuencia de colelitiasis y la gran experiencia nacional en el tratamiento de esta patología. A pesar de estar incluida entre las "Garantías explícitas en Salud" (GES) y en la cual ha habido una importante implementación y aporte de recursos para cumplir con el protocolo correspondiente, llama la atención que algunos centros públicos aún efectúen un bajo porcentaje de colecistectomías por la vía laparoscópica, la que constituye sin duda la vía de elección para este procedimiento. En cuatro de los centros encuestados, este porcentaje es menor del $60 \%$, hecho ya mencionado por Csendes ${ }^{1}$ en una serie que resume las prestaciones nacionales realizadas durante los años 2004 y 2005.

La colangiografía intraoperatoria es realizada por las distintas instituciones en un muy bajo porcentaje, debiendo ser considerada como de utilización más bien de tipo incidental. Siendo este método el patrón de oro para el diagnóstico de la coledocolitiasis, las cifras reportadas de coledocolitiasis asociada a colelitiasis no deben corresponder en estricto rigor a la verdadera frecuencia.

Lesiones iatrogénicas de vía biliar durante colecistectomía laparoscópica fue reportada en un rango de 0 a $1,5 \%$, con mediana de $0,3 \%$, cifras que son semejantes a las reportadas por otros autores nacionales ${ }^{2-5}$ en períodos anteriores, que parecen ser ya estables y que representan el riesgo inherente a esta técnica.

Esta encuesta refleja también la diferente incidencia de cáncer vesicular en colecistectomías por colelitiasis según se trate de la ubicación geográfica de la institución de salud. Mientras los centros del norte y metropolitanos reportan incidencias en torno al 3\%, dos instituciones del sur informan frecuencias sobre el $7 \%$ de cáncer.

Otra patología con clara diferencia geográfica es el quiste hidatídico hepático. El número de casos atendidos durante el año 2007 en las instituciones del centro norte y metropolitana es bastante bajo, siendo francamente distinto a las del sur del país, lo cual sigue la conocida distribución que presenta esta enfermedad, siendo las zonas ganaderas del sur las de mayor incidencia de hidatidosis.

La cirugía pancreática resectiva tiende a ser realizada más frecuentemente en centros metropolitanos, tanto para la resección cefálica como corporocaudal. Esta serie resume un número de 211 resecciones pancreáticas, cifra mayor a la reportada previamente ${ }^{1}$ para el año 2005.

El Trasplante Hepático fue reportado por cuatro centros del área privada y de la Región Metropolitana.

Esta sencilla encuesta sólo ha pretendido actualizar e ilustrar algunas de las principales cifras con respecto a las enfermedades hepato-bilio-pancreáticas que ocurren en el país. Pretendemos que, a través del Departamento de Cirugía de Hígado, Vía Biliar y Páncreas, podamos en el futuro lograr establecer canales de cooperación e información, que permitan lograr un objetivo conocimiento de nuestras cifras con respecto a este tipo de patologías, tan propias de nuestro país. Nuestro agradecimiento muy sincero a todos quienes cooperaron en la recolección y envío de la información de los diversos centros participantes.

\section{Referencias}

1. Csendes A, González G. Cirugías digestivas más frecuentes en Chile, excluyendo colorrectal. Rev Chil Cir 2008; 60: 379-386.

2. Hepp J, Ríos H. Colecistectomía laparoscópica: evaluación de 35.014 operaciones realizadas en Chile. Rev Chil Cir 1996; 48: 461-466.

3. Yarmuch J, Schutte H, Csendes A, Watkins G, Braghetto I, De La Cuadra R, y cols. Problemas y complicaciones durante la colecistectomía laparoscópica; a propósito de 2.644 operados. Rev Chil Cir 1996; 48: 375-380.

4. Hernández F, Rodríguez C, Matus C, Cerda R, Leiva L. Lesión mayor de vía biliar en colecistectomía. Rev Chil Cir 2004; 56: 16-20.

5. Coddou E. Manejo de las lesiones iatrogénicas de la vía biliar en el hospital San Pablo de Coquimbo. Rev Chil Cir 2006; 58: 12-19. 